\title{
Strategies for Oligoribonucleotide Synthesis According to the Phosphoramidite Method
}

Research in the many roles of ribonucleic acids was hindered by limited means of producing such biologically relevant molecules (Gold, 1988; Francklyn and Schimmel, 1989; Cook et al., 1991; Cech, 1992). Although enzymatic methods existed, protocols that allowed one to probe structure-function relationships were limited. Only uniform postsynthetic chemical modification (Karaoglu and Thurlow, 1991) or site-directed mutagenesis (Johnson and Benkovic, 1990) was available. In the latter case, researchers were limited to use of natural bases. Fortunately, adaptation of the phosphoramidite protocol for DNA synthesis to RNA synthesis has greatly accelerated our understanding of RNA. Site-specific introduction of modified nucleotides to any position in a given RNA has now become routine. Furthermore, one is not confined to a single modification but can include many variations in each molecule.

It is seemingly out of proportion that one small structural modification could cause such a dilemma; however, the presence of a single hydroxyl at the $2^{\prime}$-position of the ribofuranose ring has been the major reason that research in the RNA field has lagged so far behind comparable DNA studies. Progress has been made in improving methods for DNA synthesis that have enabled the production of large amounts of antisense deoxyoligonucleotides for structural and therapeutic applications. Only recently have similar gains been achieved for ribonucleotides (Sproat et al., 1995; Wincott et al., 1995; Vargeese et al., 1998).

The chasm between DNA and RNA synthesis is the result of the difficulty of identifying orthogonal protecting groups for the $5^{\prime}$ - and 2 '-hydroxyls. Historically, two standard approaches were taken by scientists attempting to solve the RNA synthesis problem: (1) developing a method that is compatible with state-ofthe-art DNA synthesis and (2) designing an approach specifically suited for RNA. Although adaptation of the DNA process provides a more universal procedure in which non-RNA amidites can easily be incorporated into RNA oligomers, the advantage to the latter approach is that one can develop a process that is best for RNA synthesis, allowing better yields to be realized. In both cases, however, similar issues are faced; for example identifying protecting groups that are compatible with synthesis conditions and yet can be removed at the appropriate juncture. This problem refers not only to the $2^{\prime}$ - and $5^{\prime}-\mathrm{OH}$ groups but also to the base- and phosphate-protecting groups. Consequently, the accompanying deprotection steps, in addition to the choice of ancillary agents, are affected. Another shared issue is the need for efficient synthesis of the monomer building blocks.

\section{BASIC CHEMISTRY OF OLIGORIBONUCLEOTIDE SYNTHESIS}

Solid-phase synthesis of oligoribonucleotides follows the same pathway as DNA synthesis (UNIT 3.3). A solid support with an attached nucleoside is subjected to removal of the protecting group on the $5^{\prime}$-hydroxyl. The incoming amidite is coupled to the growing chain in the presence of an activator. Any unreacted 5'-hydroxyl is capped, and the phosphite triester is then oxidized to provide the desired phosphotriester linkage. The process is then repeated until an oligomer of the desired length results. The actual reagents used may vary according to the 5'- and 2'-protecting groups. Other ancillary reagents may also differ.

Once the oligoribonucleotide has been synthesized, it must then be deprotected. This is typically a two-step process that entails cleavage of the oligomer from the support and deprotection of the base- and phosphate-blocking groups, followed by removal of the 2 '-protecting groups. Occasionally, a different order of reactions or separate deprotection of the phosphate groups is required. In all cases, it is imperative that indiscriminate removal of protecting groups not occur; this is particularly an issue in the classic situation wherein the first step is base mediated. In this case, if the 2'-hydroxyl is revealed under these conditions, strand scission will result because of attack of the vicinal hydroxyl group on the neighboring phosphate backbone (UNIT 2.2). Two other concerns that are prevalent in RNA synthesis but play no part in DNA synthesis are the propensity for $\left(3^{\prime} \rightarrow 2^{\prime}\right)$-phosphodiester migration to provide undesired $\left(2^{\prime} \rightarrow 5^{\prime}\right)$-linkages and the susceptibility of oligoribonucleotides to degradation by ribonucleases. The latter fact has led many researchers to develop 2'-protecting 
groups that can remain in place until the oligomer is required for the desired experiment. Obviously, the parameters of $2^{\prime}$-deprotection are dictated by the protecting groups used; they will be discussed in the appropriate sections.

\section{SOLID SUPPORTS}

As in DNA synthesis, the solid-phase synthesis of RNA by the phosphoramidite approach requires that one start with a solid support that is functionalized with the appropriate nucleoside corresponding to the $3^{\prime}$-end of the desired oligoribonucleotide. Typically, the nucleoside is attached to the support through a succinate linkage that is cleaved under alkaline conditions at the end of the synthesis (UNITS 3.1 \& 3.2). Controlled-pore glass (CPG; Pon et al., 1988; Damha et al., 1990) and polystyrene (McCollum and Andrus, 1991) are the most commonly used solid supports for the synthesis of RNA. There have been reports that polystyrene resins are optimal for the synthesis of RNA on small scales, $<50 \mu \mathrm{mol}$ (Sproat et al., 1995; Wincott et al., 1995); however, recent literature describes excellent syntheses of RNA on CPG on scales of $\geq 100 \mu \mathrm{mol}$ (Vargeese et al., 1998). In both cases, the best results are obtained with loadings of $\sim 30 \mu \mathrm{mol} / \mathrm{g}$.

\section{ACTIVATION OF RIBONUCLEOSIDE PHOSPHORAMIDITES}

A drawback to solid-phase RNA synthesis has been the coupling step. (See Fig. 3.5.1 for the activators discussed below.) An activator reacts with the incoming amidite to produce a reactive electrophilic intermediate that is attacked by the $5^{\prime}$-hydroxyl of the growing polymer chain. Because of the usually bulky $2^{\prime}$-protecting group, coupling reactions between ribonucleoside phosphoramidites are typically sluggish. Reaction times of as much as $1 \mathrm{hr}$ have been reported, although incubation times of anywhere from 10 to $30 \mathrm{~min}$ have usually been achieved with $1 H$-tetrazole (S.1) as the activator (Usman et al., 1987; Scaringe et al., 1990). This is in contrast to the extremely short coupling times required for DNA, typically on the order of $30 \mathrm{sec}$. Furthermore, coupling is usually not as efficient as observed with DNA, average stepwise yields (ASY) of $97 \%$ are common, whereas ASY of $99 \%$ are regularly achieved with DNA. Changes in concentration and/or coupling time result only in additional side products, not increased coupling yields.

Recently the use of substituted $1 \mathrm{H}$-tetrazoles (Leiber and Enkoju, 1961) as activators for DNA and RNA synthesis was reported (Andrus et al., 1986; Vinayak et al., 1994). 5Ethylthio-1 $H$-tetrazole (S.2) was found to be a more effective activator than $1 H$-tetrazole because of its higher solubility in acetonitrile and greater acidity (Sproat et al., 1995; Wincott et al., 1995). There are also a number of reports of the use of 5-(3-nitrophenyl)-1H-tetrazole (S.3) as an effective activator for RNA synthesis. Coupling times as short as $6 \mathrm{~min}$ have been reported (Rao et al., 1993). Two additional activators were recently described in the literature: 4,5-dicyanoimidazole (S.4; Vargeese et al., 1998) and benzimidazolium triflate (S.5; Hayakawa et al., 1996). These reagents rely on increased nucleophilicity to enhance the rate of the coupling reaction without increasing acidity.

It should also be emphasized that choosing the appropriate protecting groups is the key to successful oligoribonucleotide synthesis. Although the 2 '- and 5'-protecting groups will be discussed in depth, one cannot ignore the importance of the base- and phosphate-blocking groups. The interplay between the protecting groups is crucial. Some base- and phosphateblocking groups are not stable to the conditions required for the repetitive removal of the $5^{\prime}$ blocking group during each nucleotide addition cycle. In other cases, the protecting group may not be stable to the conditions required to produce the monomers. Specific cases will be discussed in this unit; however, generic protection strategies are delineated below.

\section{NUCLEOBASE-PROTECTING GROUPS}

Standard DNA nucleobase-protecting groups (benzoyl for $\mathrm{A}$ and $\mathrm{C}$ and isobutyryl for G) can be easily removed by treatment with

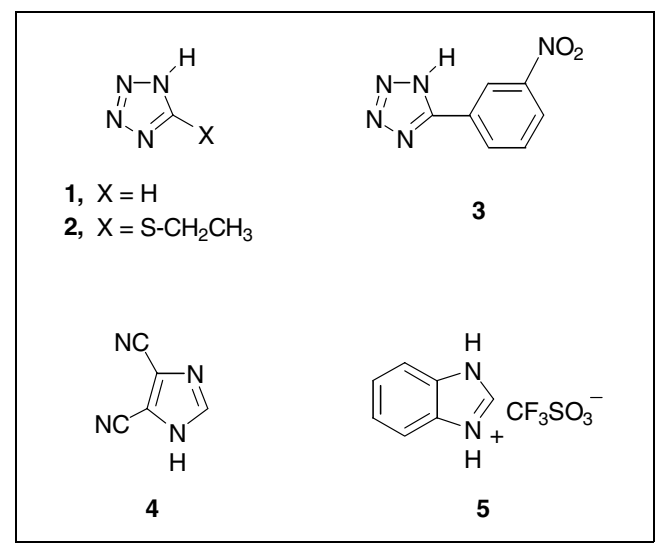

Figure 3.5.1 Activators commonly used for the synthesis of oligoribonucleotides. 
concentrated $\mathrm{NH}_{4} \mathrm{OH}$. Many of the 2'-protecting groups used in RNA synthesis, however, are unstable to this harsh reagent. Although milder deprotection conditions have been developed- $\mathrm{NH}_{4} \mathrm{OH} / \mathrm{EtOH}(3 / 1, \mathrm{v} / \mathrm{v}$; Usman et al., 1987) or $\mathrm{EtOH} / \mathrm{NH}_{3}$ (Scaringe et al., 1990)long incubation times are still required $(\sim 16 \mathrm{hr}$ at $55^{\circ} \mathrm{C}$ to $65^{\circ} \mathrm{C}$ ). Consequently, protecting groups with increased base sensitivity were developed: phenoxyacetyl (S.6; Wu et al., 1988), (4-isopropylphenoxy)acetyl (S.7), (4-tbutylphenoxy)acetyl (S.8; Chaix et al., 1989; Sinha et al., 1993), and $N, N$-dialkylformamidines (S.9; Theisen et al., 1993). See Figure 3.5.2 for the structures. These nucleobase-protecting groups can be removed by treatment with $\mathrm{NH}_{4} \mathrm{OH} / \mathrm{EtOH}(3 / 1, \mathrm{v} / \mathrm{v})$ within $4 \mathrm{hr}$ at $55^{\circ} \mathrm{C}$. Because of this short deprotection protocol, better yields of higher-quality RNA product were obtained. An even faster deprotection method for synthetic RNA oligonucleotides was recently reported (Reddy et al., 1995; Wincott et al., 1995). The method entails the use of aqueous methylamine at $65^{\circ} \mathrm{C}$, which reduces oligonucleotide deprotection time to $10 \mathrm{~min}$ and produces full-length product in yields higher than those obtained with the standard $\mathrm{NH}_{4} \mathrm{OH} / \mathrm{EtOH}(3 / 1, \mathrm{v} / \mathrm{v})$ deprotection protocol (Wincott et al., 1995). With this reagent, however, the cytosines of any given oligonucleotide must by N-protected with an acetyl group to prevent transamination during deprotection (Reddy et al., 1994).

Another family of nucleobase-protecting groups for RNA synthesis relates to the 2-(4nitrophenyl)ethyl (Npe) S.10 and 2-(4-nitrophenyl)ethoxycarbonyl (Npeoc) $\mathbf{S . 1 1}$ groups (Himmelsbach et al., 1984). They are stable to both weak acids and weak bases and yet can be readily removed with a non-nucleo-

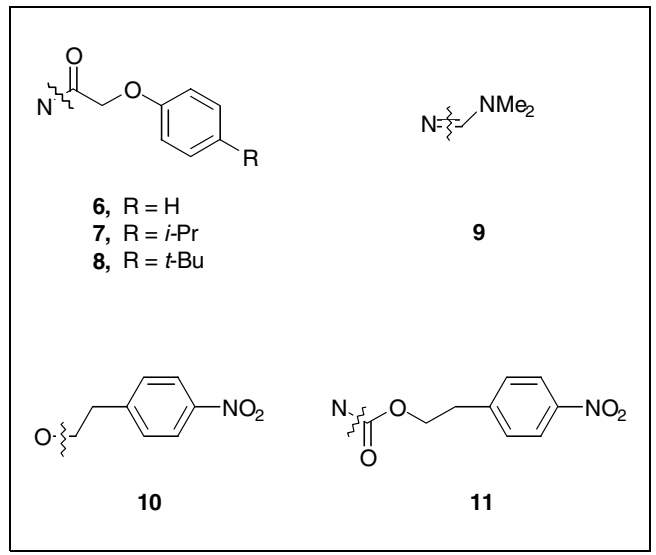

Figure 3.5.2 Base-protecting groups typically used for the synthesis of oligoribonucleotides. philic base such as 1,8-diazabicyclo[5.4.0]undec-7-ene (DBU). The use of these $\beta$-eliminating blocking groups is usually indicated when an acid-labile $5^{\prime}$-protecting group is present.

\section{PHOSPHATE PROTECTION}

Another approach to optimizing the coupling step in RNA synthesis has been to modify either the dialkylamine or the protecting group component of the ribonucleoside phosphoramidite function. In most cases, the 2-cyanoethyl $/ N, N$-diisopropylamino combination is used for the synthesis of oligoribonucleotides. The use of lower-alkyl-substituted phosphoramidites, such as diethylamino, instead of diisopropylamino has been reported to improve coupling yields in RNA synthesis when measured by dimethoxytrityl cation quantitation (Lyttle et al., 1991). These compounds, however, have not been used extensively because of their instability.

In regard to the phosphate-protecting groups, the 2-cyanoethyl is most favored; however, in some instances other protecting groups must be employed. For example, different phosphate-protecting groups may be required because of incompatibility of the 2-cyanoethyl group with synthesis or deprotection conditions or for increasing coupling rates by offsetting the bulky 2 '-protecting group with a smaller phosphorous moiety. In these cases, the Npe (Himmelsbach et al., 1984) or methyl (Usman et al., 1985) group may advantageously replace the 2-cyanoethyl group for phosphate protection. The selection of phosphate protecting groups along with appropriate deprotection conditions will be discussed on a case-by-case basis in conjunction with the groups being used for $5^{\prime}$ and $2^{\prime}$ protection.

Now that the general parameters regarding the synthesis of oligoribonucleotides have been reviewed, specific synthetic strategies that affect the choice of 5'- and 2'-protecting groups will be discussed.

\section{2'-HYDROXYL PROTECTION}

The most common paradigm has been to adapt DNA synthesis to the preparation of RNA oligonucleotides. As a result, a 2'-hydroxylprotecting group must be identified that is compatible with DNA-protecting groups and easily be removed once the oligomer is synthesized (UNIT 2.2). See Figure 3.5.3 for the 2'-hydroxylprotecting groups discussed below. Owing to constraints placed by the existing amide-protecting groups on the bases and the $5^{\prime}$-O - dimethoxytrityl (DMTr) group-or in some
Synthesis of Unmodified Oligonucleotides 


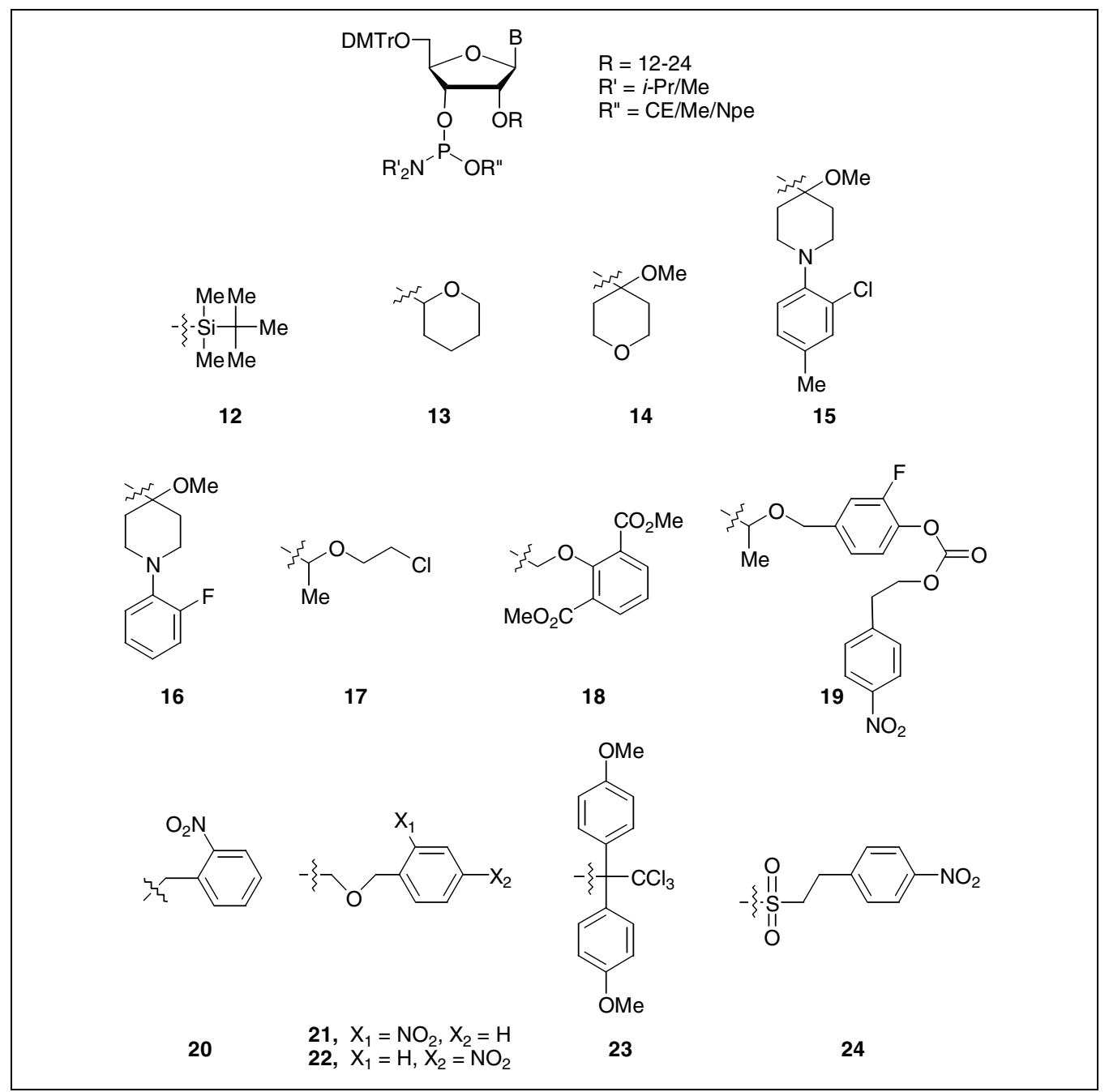

Figure 3.5.3 2'-Protecting groups that are compatible with solid-phase oligonucleotide synthesis protocols.

cases the 5-O-(9-(phenyl)xanthen-9-yl (Px) group-the 2'-blocking group must be stable to both acid and base. In addition, the group must also be inert to the oxidizing and capping reagents. Although the most widely used 2'-hydroxyl-protecting group is the tert-butyldimethylsilyl (TBDMS) group, many others have been explored because of the longer coupling times required when the bulky $2^{\prime}-O$-TBDMS substituent is used. Other types of $2^{\prime}$ protection will be covered in the following sections (a number of these can also be found in Gait et al., 1991, and Beaucage and Iyer, 1992).

\section{The TBDMS Group}

By far the most popular 2'-protecting group is the TBDMS (S.12), developed principally by Ogilvie and co-workers (Usman et al., 1987). Synthesis of 2'-O-TBDMS nucleoside derivatives can be quite readily accomplished in good yields (Scaringe et al., 1990). The chemistry used in the construction of oligoribonucleotides is completely compatible with the DNA synthesis cycle, thereby allowing for the simple preparation of DNA/RNA chimeras. In addition, many of the earlier disadvantages of the TBDMS group no longer exist. Although amidite coupling times are not as short as with DNA, through the use of 5-ethylthio- $1 H$-tetrazole (S.2), coupling times have been reduced from $30 \mathrm{~min}$ to as low as $5 \mathrm{~min}$ (Wincott et al., 1995). Furthermore, new deprotection protocols have not only reduced incubation times but also greatly increased the quality of the product to the extent that contaminating oligonucleotides with $\left(2^{\prime} \rightarrow 5^{\prime}\right)$ phosphodiester linkages can be eliminated completely.

Deprotection of oligoribonucleotides containing $2^{\prime}-O$-TBDMS groups was once a twostep process that first entailed a basic step similar to that used for the deprotection of DNA oligonucleotides, in which an oligomer was 
cleaved from the support and the base and phosphate groups were removed. As stated earlier, through the use of methylamine this step has been reduced to $10 \mathrm{~min}$. The second step was the removal of the $2^{\prime}-O$-TBDMS groups from the oligonucleotide. In the past, this was accomplished by treatment with $1 \mathrm{M} n$ tetrabutyl ammonium fluoride (TBAF) in tetrahydrofuran (THF) at room temperature over 24 hr (Usman et al., 1987; Scaringe et al., 1990). Unfortunately, the use of this deprotecting agent produces salts that must be removed before analysis and purification. In addition, the long exposure time required for complete removal of the protecting group, coupled with the reagent's sensitivity to adventitious water (Hogrefe et al., 1994), made it less than ideal. Although a few reports were published regarding the use of neat triethylamine trihydrofluoride (TEA $\bullet$ HF) as a desilylating reagent (Gasparutto et al., 1992; Westman and Strömberg, 1994), results were mixed. A solution of TEA $\bullet 3 \mathrm{HF}$ in $N$-methyl pyrrolidinone (NMP; Wincott et al., 1995) or N,N-dimethylformamide (DMF; Sproat et al., 1995) has also been described from which full deprotection can be achieved in 30 to $90 \mathrm{~min}$ at $65^{\circ} \mathrm{C}$ or 4 to $8 \mathrm{hr}$ at room temperature. As an added advantage, because no salts are produced, the product can be directly precipitated from the desilylating reagent. More recently, a further improved procedure was reported in which both the basic deprotection and desilylation reaction can be accomplished in one pot using a mixture of methylamine in ethanol followed by the addition of TEA $\bullet 3 \mathrm{HF}$ (Bellon, 1999). This protocol allows for the complete deprotection of an oligoribonucleotide in $<2 \mathrm{hr}$ without any evidence of $\left(3^{\prime} \rightarrow 2^{\prime}\right)$-phosphodiester migration.

\section{Acetal-Protecting Groups}

Because of concerns about conversion of the desired $\left(3^{\prime} \rightarrow 5^{\prime}\right)$-internucleotidic linkages to $\left(2^{\prime} \rightarrow 5^{\prime}\right)$ linkages, acid-labile acetals were thought to be the ideal 2'-protecting groups. They are stable to alkaline conditions and can be hydrolyzed with dilute acids; therefore, there are no residual reagents to complicate purification. Furthermore, the oligonucleotide can be isolated with the 2 '-protecting group intact, thereby allowing one to store the oligonucleotide in a nuclease-resistant form.

A number of different acetals have been investigated. The 2'-O-tetrahydropyranyl (Thp) S.13 and 2'-O-methoxytetrahydropyranyl (Mthp) $\mathbf{S . 1 4}$ groups proved to be unstable to the conditions required for iterative removal of the 5'-O-DMTr group (Reese and Skone, 1985; Christodoulou et al., 1986). Although some successful syntheses have resulted from the use of these acetals, they have been limited to very short oligomers. As a result, aryl-substituted piperidines were developed. The 2'-O-[1-(2-chloro-4-methylphenyl)4-methoxypiperidin-4-yl] (Ctmp) group (S.15) and the 2'-O-[1-(2-fluorophenyl)-4-methoxypiperidin-4-yl] (Ftmp) group (S.16) were first investigated by Reese and co-workers (1986; UNIT 2.2). The Fpmp group is relatively easy to prepare and readily incorporated into ribonucleosides to provide the required phosphoramidite monomers. The $2^{\prime}$ - $O$-Fpmp group is more stable to acidic hydrolysis than $2^{\prime}-O$ Thp and 2'-O-Mthp acetals (Beijer et al., 1990) and can, therefore, be used in conjunction with either a $5^{\prime}$-O-DMTr or a $5^{\prime}$-O-Px group in solid-phase RNA synthesis. Coupling times of 3 to $12 \mathrm{~min}$ have been reported for ribonucleoside 2'-O-Fpmp phosphoramidite derivatives upon activation with nitrophenyl-substituted $1 H$-tetrazoles (Beijer et al., 1990; Rao et al., 1993; Capaldi and Reese, 1994). Early reports indicated that deprotections of $2^{\prime}-O$ Fpmp oligonucleoties was optimal at $\mathrm{pH} 2.0$ for $20 \mathrm{hr}$ at room temperature (Rao et al., 1993). It has since, however, been determined that the rate of acid-catalyzed hydrolysis of internucleotidic linkages is sequence dependent. To avoid hydrolytic cleavage and phosphodiester migration, the removal of the 2'-O-Fpmp groups should be performed at a $\mathrm{pH}$ above 3.0 for $24 \mathrm{hr}$ at room temperature (Capaldi and Reese, 1994; UNIT 2.2).

Another approach to acetal protection of the 2 -hydroxy function led to the development of the 1-(2-chloroethoxy)ethyl group (S.17; Sakatsume et al., 1991a,b). Oligoribonucleotides of up to 20 residues in length have been prepared using $\mathbf{S . 1 7}$ for 2'-OH protection. This protecting group is stable under the acidic conditions required for removing the $5^{\prime}-O$ DMTr group and yet can be removed postsynthetically within $30 \mathrm{hr}$ upon hydrolysis with $0.01 \mathrm{~N} \mathrm{HCl}(\mathrm{pH} 2.0)$ at room temperature. No base modification or phosphodiester migration was detected. In light of the results of Capaldi and Reese (1994), deprotection at pH 3.0 might be worth investigating. Furthermore, synthesis of the 2'-O-protected ribonucleosides proceeds quite smoothly from the corresponding Markiewicz-protected nucleosides.

More recently, a new 2 -protecting group was reported: the 2-hydroxyisophthalate for-
Synthesis of Unmodified Oligonucleotides 
maldehyde acetal (S.18; Rastogi and Usher, 1995). This is a convertible protecting group that, as the diester, is stable to acidic treatment during synthesis but is converted upon treatment with ammonia to a diacid that is more labile under acidic conditions than the parent diester. The half-life for the deprotection of the resulting diacid is $\sim 390 \mathrm{~min}$ at $\mathrm{pH} 3$ compared to $166 \mathrm{~min}$ for the cleavage of $\mathbf{S . 1 6}$ under the same conditions. At this time only UpU and UpG dimers and the corresponding uridine phosphoramidite have been synthesized.

Finally, Pfleiderer et al. (1996) designed a new acetal for the solid-phase synthesis of oligoribonucleotides that is used successfully in conjunction with a $5^{\prime}-O$-DMTr group. Like the 2 -protecting group $\mathbf{S . 1 8}$, the $2^{\prime}-O$-acetal, $1-\{4$ [2-(4-nitrophenyl)ethoxycarbonyloxy]-3fluorobenzyloxy \}ethyl (S.19), is a convertible, or "protected protecting," group. Cleavage of the (4-nitrophenyl)ethoxycarbonyl group from S.19 results in a $2^{\prime}-O$-acetal that can be hydrolyzed within $4 \mathrm{hr}$ under acidic conditions; this is in sharp contrast to the $24 \mathrm{hr}$ required for hydrolysis of the parent $2^{\prime}$ - $O$-acetal $\mathbf{S . 1 9}$ under the same conditions. Ribonucleoside phosphoramidites functionalized with the $2^{\prime}-O$ acetal S.19 and Npe/Npeoc (S.10/S.11) baseprotecting groups require a $20 \mathrm{~min}$ coupling time for optimal solid-phase RNA synthesis. Oligoribonucleotides were treated with DBU to remove base- and phosphate-protecting groups as well as the (4-nitrophenyl)ethoxycarbonyl group of the 2'-O-acetal S.19. After cleavage from the support, the deprotected 2'$O$-acetal was cleaved from RNA oligomers upon acidification with $0.5 \% \mathrm{AcOH}$ for $18 \mathrm{hr}$ at room temperature. The use of $80 \% \mathrm{AcOH}$ $(\mathrm{pH}<3)$ led to $\left(3^{\prime} \rightarrow 2^{\prime}\right)$-phosphodiester migration along with strand scission (Capaldi and Reese, 1994).

\section{Photolabile Groups}

Another approach to the protection of the ribonucleoside 2 '-hydroxyl is the use of photolabile-protecting groups. This strategy has many advantages. The protecting groups are completely orthogonal, because they are resistant to both acid and base and, as a result, remain intact throughout synthesis and final deprotection. Furthermore, incorporation of such protecting groups into ribonucleosides is accomplished quite readily without any migration. Originally, the $2^{\prime}-O$-(o-nitrobenzyl) group (S.20) was the photolabile-protecting group of choice (Ohtsuka et al., 1981; Hayes et al., 1985). Coupling of the corresponding amidite was accomplished in 15 min with tetrazole as the activator or $2.5 \mathrm{~min}$ using 5-( $p$-nitrophenyl) $1 H$-tetrazole in conjunction with the methyl phosphate-protecting group (Tanaka et al., 1986). After oligonucleotide deprotection under basic conditions, the remaining $2 '-O$-(o-nitrobenzyl) groups were cleaved upon irradiation of the oligomer with longwave UV light at $\mathrm{pH} 3.5$ for $1 \mathrm{hr}$ in solutions that have been purged with $\mathrm{N}_{2}$. At higher $\mathrm{pH}$, the formation of side products occurred.

Because the 2'-O-(o-nitrobenzyl) group (in conjunction with the 2-cyanoethyl phosphate group) requires extended amidite coupling times (deBear et al., 1987), the $o$-nitrobenzyloxymethyl (S.21) group was proposed as an alternative 2 -photolabile-protecting group (Schwartz et al., 1992). It was postulated that the extended arm present in this group might ease steric crowding, thereby reducing amidite coupling times. Synthesis of the corresponding ribonucleoside phosphoramidite monomers proceeds similarly to that of the $2^{\prime}-O$-TBDMS amidites. Unlike silyl-protected ribonucleotides, however, these amidites required only a 2-min coupling time. After a standard basic deprotection protocol-pyridine: $\mathrm{NH}_{4} \mathrm{OH}(1: 4, \mathrm{v} / \mathrm{v}), 50^{\circ} \mathrm{C}, 24 \mathrm{hr}$ - the $2^{\prime}$ protected oligomers were exposed to longwave UV light at $\mathrm{pH} 3.7$ for $4.5 \mathrm{hr}$ at room temperature to remove the $o$-nitrobenzyloxymethyl groups. More recently, the $p$-nitrobenzyloxymethyl (S.22) group was recommended for 2'-OH protection (Gough et al., 1996). This protecting group behaves almost identically to $\mathbf{S . 2 1}$ in regard to amidite monomers synthesis and coupling times; however, it can be removed from 2 '-protected oligoribonucleotides within $24 \mathrm{hr}$ upon reaction with TBAF at room temperature.

\section{The 1,1-Dianisyl-2,2,2-Trichloroethyl Group}

Klosel et al. (1996) described a completely new protecting group for $2^{\prime}$ protection of ribonucleosides: the 2'-O-1,1-dianisyl-2,2,2trichloroethy group (S.23). This $\beta$-haloalkyl group is stable to acid and base and yet is cleavable under mild, neutral conditions via reductive fragmentation. Furthermore, there is no migration after the protecting group between the $2^{\prime}$ - and the $3^{\prime}$-hydroxyls. Only synthesis of the uridine phosphoramidite and the corresponding UpT dimer has been described. Synthesis of the amidite monomer is fairly straightforward; the product is prepared in five steps from uridine. A 15-min 
coupling time is required for the activated amidite to form the dimer. After treatment with ammonia, the dimer was exposed to lithium cobalt(I)phthalocyanine and phenol in $\mathrm{MeOH}$ $\left(\mathrm{O}_{2}\right.$ free $)$ for $14 \mathrm{hr}$ at room temperature to effect cleavage of the $2^{\prime}$-protecting group. The reaction mixture was then quenched by the addition of buffer; upon analysis it was shown that the backbone was intact and that no $\left(2^{\prime} \rightarrow 5^{\prime}\right)$-phosphodiester linkage was present.

\section{The $p$-Nitrophenylethyl Sulfonyl}

\section{Group}

The $p$-nitrophenylethyl sulfonyl group (S.24) has also been proposed as a 2 '-protecting group for ribonucleosides (Pfister et al., 1988). The advantages of this sulfonate-derived group are acid stability and the absence of $\left(2^{\prime} \rightarrow 3^{\prime}\right)$ migration. This protecting group works best when the Npeoc and 2-cyanoethyl groups are used for base and phosphate protection, respectively. Treatment of 2'-protected oligonucleotides with DBU results in the removal of all protecting groups. Unfortunately, this $2^{\prime}$ protection strategy is not compatible with uridine that is unprotected at O4 because of concomitant anhydro nucleoside formation. As a result, protection of $\mathrm{O} 4$ with a 2-cyanoethyl group was explored. This group can also be removed upon exposure to DBU, however only at elevated temperatures $\left(50^{\circ} \mathrm{C}\right)$.

\section{ALTERNATIVE TO 5'-TRITYL DERIVATIVES FOR THE $5^{\prime}$-OH PROTECTION OF RIBONUCLEOSIDES}

In many cases, researchers have chosen a de novo approach to the synthesis of oligoribonucleotides in which the focus is not on develop-

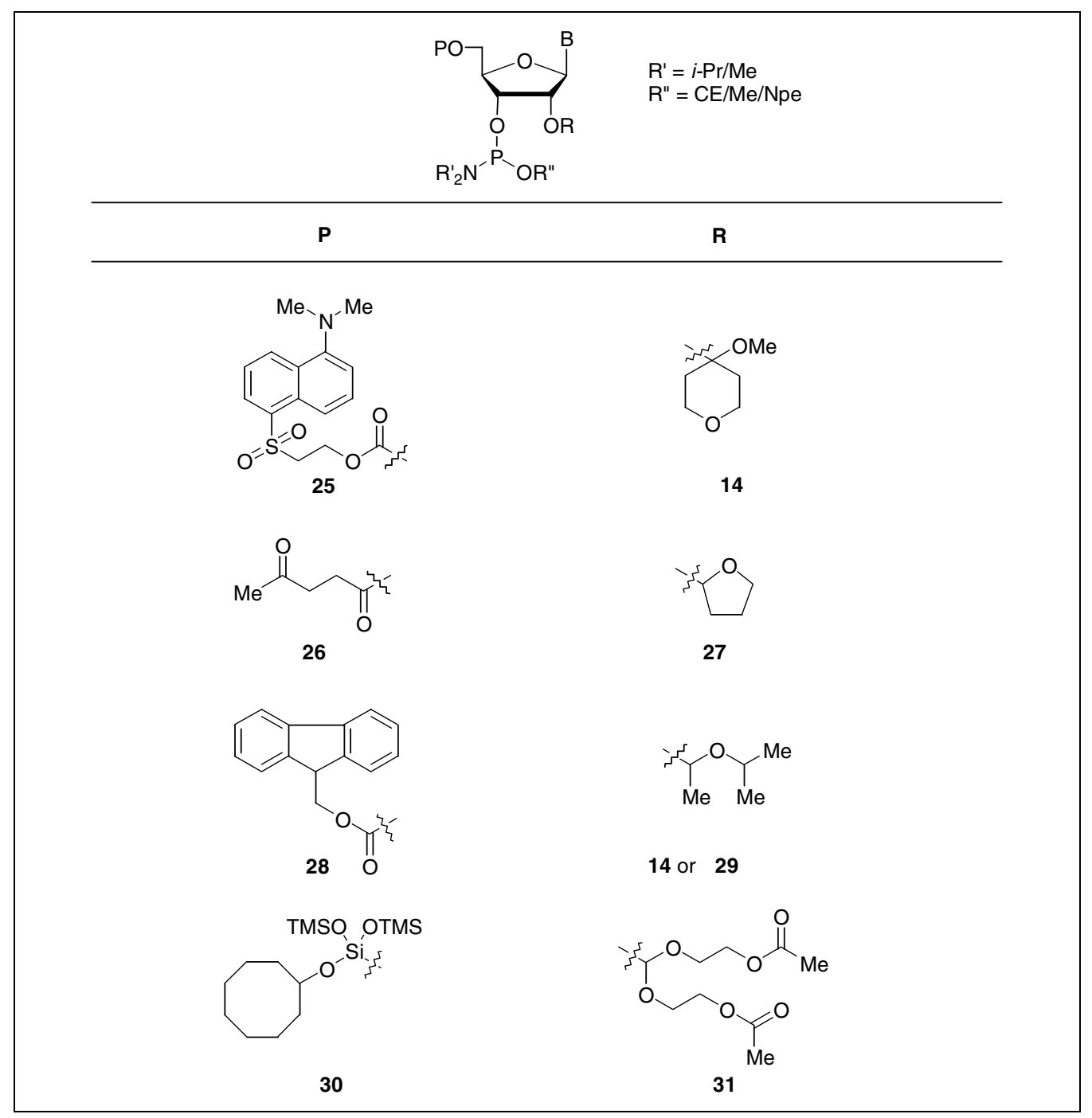

Figure 3.5.4 $5^{\prime}(\mathrm{P})$ - and $2^{\prime}(\mathrm{R})$-protecting group combinations for the synthesis of oligoribonucleotides.
Synthesis of Unmodified Oligonucleotides 
ing a method that is compatible with DNA synthesis but rather evolving a process that is best for RNA synthesis. A number of different approaches have been explored. In most cases, the decision was made to proceed with an acetal-protecting group for 2'-protection. This choice was made for all the reasons stated earlier: there is no $\left(2^{\prime} \rightarrow 3^{\prime}\right)$ migration during amidite monomer synthesis; no residual reagents are present after deprotection; and oligomers can be stored with the 2 '-protecting groups in place until the RNA product is needed, thereby protecting it against nuclease degradation. Because these groups are acid labile, the $5^{\prime}$-protecting groups that have been developed are typically base labile or sensitive to fluoride ions. Obviously, these constraints further affect the choice of suitable nucleobases and phosphate-protecting groups. Some of the options that have been explored are presented in this unit (see Fig. 3.5.4).

\section{The 2-Dansylethoxycarbonyl Group}

One approach to resolving the incompatibility of $2^{\prime}-O$-acetal-protecting groups with the standard acid-labile 5'-O-DMTr and 5'-O-Px groups in oligoribonucleotide synthesis was the development of the 2-dansylethoxycarbonyl group for 5' protection (S.25; Bergmann and Pfleiderer, 1994a). This base-labile group can be readily removed (in $140 \mathrm{sec}$ ) using dilute DBU (Bergmann and Pfleiderer, 1994b). As a result, the more stable 2-(4-nitrophenyl)ethyl phosphate-protecting group is required in place of the traditional 2-cyanoethyl group; Npe (S.10) and Npeoc (S.11) are used for base protection. When this protecting group scheme was used in conjunction with a $2^{\prime}-O$-Mthp group, the coupling reaction time of the corresponding phosphoramidite was 12 min with $1 \mathrm{H}$-tetrazole. Oligomers of 20 nucleotides were synthesized; and in special cases, polymers of 40 nucleotides were prepared. It was determined that the best results were obtained with the $N, N$-diethylphosphoramidite rather than with the $N, N$-diisopropyl analog. Deprotection of the oligoribonucleotide first required a 10-hr treatment with 0.5 to $1 \mathrm{M} \mathrm{DBU}$ to remove the nucleobase- and phosphate-protecting groups, followed by cleavage from the support by ammonolysis $(200 \mathrm{~min})$. The oligomer could be stored at this point or exposed to acid to remove the $2^{\prime}$-O-Mthp group (S.14).

\section{The Levulinyl Group}

Oligoribonucleotides (21-mers) were synthesized using a $5^{\prime}-O$-levulinyl $(\mathbf{S . 2 6}) / 2^{\prime}-O$ - tetrahydrofuranyl (S.27) protecting group combination (Iwai et al., 1987; Iwai and Ohtsuka, 1988). The $5^{\prime}$-O-levulinyl group is removed during solid-phase synthesis by hydrozinolysis. After ammonia treatment, the base- and phosphate-deprotected oligomer is then treated with $0.01 \mathrm{~N} \mathrm{HCl}(\mathrm{pH} 2)$ for $24 \mathrm{hr}$ to effect removal of the $2^{\prime}-O$-acetal. Although, no base modification is observed, there are some drawbacks to this scheme. As in the case of a number of 5'-protecting groups, removal of the levulinyl group cannot be monitored. Furthermore, because of the prolonged amount of time required for full removal of the $5^{\prime}$ - $O$-levulinyl group, cycle times are very long. Finally, introduction of the levulinyl group to the $5^{\prime}$-position of ribonucleosides is not selective, thereby reducing yields.

\section{The 9-Fluorenylmethoxycarbonyl Group}

The lability of acetal groups to iterative acidic treatment led to the development of the 9-fluorenylmethoxycarbonyl (Fmoc) group (S.28) as an alternative to the DMTr group for 5'-hydroxyl protection (Lehmann et al., 1989). The Fmoc group is readily introduced to the $5^{\prime}$-position of the 2'-O-Mthp-protected nucleosides. During solid-phase oligonucleotide synthesis, the 5'-O-Fmoc group is removed before each chain elongation step by a brief treatment (2 min) with $0.1 \mathrm{M}$ DBU in acetonitrile. The release of the Fmoc group can be monitored by UV spectroscopy, thereby allowing quantitation of the ribonucleoside phosphoramiditecoupling efficiency. The coupling reaction time of incoming amidites activated by $5-(p$ nitrophenyl)- $1 H$-tetrazole ranges between 2 and $10 \mathrm{~min}$. Upon completion of the synthesis, the oligoribonucleotide is treated with ammonia to remove nucleobase- and phoshate-protecting groups and release it from the support. At this point, the 2'-protected oligoribonucleotide can be purified, if so desired. Deprotection of the 2'-O-Mthp groups takes place at $\mathrm{pH} 2.0$ within $4 \mathrm{hr}$ at room temperature. After careful analysis, it was shown that all internucleotidic linkages were $\left(3^{\prime} \rightarrow 5^{\prime}\right)$ and that no base modification occurred. Oligomers of up to 20 residues have been successfully synthesized using this combination of protecting groups.

Ogawa et al. (1991) substituted the acidlabile 1-(isopropoxy) ethyl (IPE) group (S.29) for the $2^{\prime}$-O-Mthp group. The desired nucleosides were prepared from the corresponding Markiewicz-protected intermediates in a fourstep procedure in good yields. Removal of the 
5'-O-Fmoc group during solid-phase synthesis was accomplished with $0.1 \mathrm{M}$ piperidine in acetonitrile ( $2 \mathrm{~min}$ ), whereas amidite coupling was effected with $1 H$-tetrazole over a period of 20 to $25 \mathrm{~min}$. Oligonucleotide deprotection consisted of a treatment with ammonia for 6 to $12 \mathrm{hr}$ at $55^{\circ} \mathrm{C}$; the $2^{\prime}$ - $O$-IPE group was removed last at $\mathrm{pH} 2.0$ within $3 \mathrm{hr}$ at room temperature. Again, no $\left(2^{\prime} \rightarrow 5^{\prime}\right)$-phosphodiester isomerization or base modification was observed under these conditions. Oligomers up to 21 residues were reported using this combination of protecting groups.

\section{The Bis(trimethylsiloxy) cyclooctyloxysilyl Group}

A completely different approach to $5^{\prime}$-protection was taken by Scaringe et al. (1998), wherein a $5^{\prime}-O$-silyl ether was used in tandem with a $2^{\prime}$ - $O$-orthoester. The $5^{\prime}$-O $O$-bis(trimethylsiloxy)cyclooctyloxysilyl ether (SIL; S.30) can be removed by fluoride ion treatment under conditions that will not affect an acid-labile $2^{\prime}$-protecting group. The $2^{\prime}$ - $O$-bis $(2$-acetoxyethoxy) methyl orthoester (ACE; S.31) is a convertible protecting group that is stable to all synthesis conditions but is modified during nucleobase deprotection under basic conditions. The resulting 2'-O-bis(2-hydroxyethoxy)methyl orthoester is 10 times more acid labile than the original orthoesterprotecting group. The $2^{\prime}-O$-protected ribonucleosides can be produced in four steps from the Markiewicz-protected nucleosides in overall yields of $45 \%$ to $55 \%$. Because 2-cyanoethyl groups are not compatible with repeated exposure to fluoride ion, methyl $N, N$-diisopropylphosphoramidite derivatives are used. The $5^{\prime}$ $O$-silyl group is removed in $35 \mathrm{sec}$ upon reaction with 1.1 M HF in TEA/DMF. Amidite coupling is complete after $90 \mathrm{sec}$ when 5ethylthio- $1 H$-tetrazole $(\mathbf{S . 2})$ is used as the activator; coupling yields were reported as $>99 \%$. Once the oligomer has been synthesized, deprotection of the methyl phosphate group is effected by disodium-2-carbamoyl-2-cyanoethylene-1,1-dithiolate (10 min;- Dahl et al., 1990); followed by treatment with aqueous $40 \%$ methylamine at $55^{\circ} \mathrm{C}$ for $10 \mathrm{~min}$. The $2^{\prime}$-protected oligomer can then be analyzed; purified, if necessary; and then stored. To remove the modified $2^{\prime}$ - $O$-orthoester, the oligoribonucleotide is heated to $55^{\circ} \mathrm{C}$ for $10 \mathrm{~min}$ in a $\mathrm{pH} 3$ buffer, followed by incubation at $\mathrm{pH} 7.7$ to 8.0 for $10 \mathrm{~min}$ at $55^{\circ} \mathrm{C}$. This final step cleaves any remaining $2^{\prime}-O$-formyl groups that result from the orthoester deprotection. Syntheses of oligomers of up to 36 residues in length have been reported. Careful analysis of the deprotected oligomers showed there was no base modification and no sign of $\left(2^{\prime} \rightarrow 5^{\prime}\right)$-phosphodiester migration. Furthermore, appropriate molecular weights and enzymatic activity were observed for the oligomers that were synthesized. It should be noted that when oligoribonucleotides are identically produced by either $5^{\prime}-O$-DMTr $/ 2^{\prime}-O$-TBDMS or the $5^{\prime}-O$ $\mathrm{SIL} / 2^{\prime}-O$-ACE phosphoramidite method, better yields of RNA oligonucleotides were obtained with the $5^{\prime}-O-\mathrm{SIL} / 2^{\prime}-O$-ACE phosphoramidite protocol.

\section{SUMMARY}

Significant advances in RNA biology and biochemistry can be achieved only through concomitant advances in RNA chemistry. The current state of the art in ribozyme research would not have been possible without the recent improvements in RNA synthesis. The current technology, however, is still limiting. There is no report of routine syntheses of tRNAs or even hairpin ribozymes. Until RNA synthesis chemistry can provide oligoribonucleotides as readily as DNA, the search for new and better methods for the synthesis of RNA will continue.

Currently, the 5'-O-DMTr$/ 2^{\prime}-O$-TBDMS is the benchmark for the synthesis of oligoribonucleotides (Usman et al., 1987; Scaringe et al., 1990; Sproat et al., 1995; Wincott et al., 1995). The use of the TBDMS-protecting group (S.12) was first described in the 1970s. In the ensuing years, many other methods for the synthesis of RNA were developed, but none has gained the popularity of the TBDMS chemistry. Recent advances in the use of this silyl chemistry in terms of synthesis (Sproat et al., 1995; Wincott et al., 1995; Vargeese et al., 1998) and deprotection (Sproat et al., 1995; Wincott et al., 1995; Bellon, 1999) have made it an even more viable approach to the production of oligoribonucleotides. In the early $1990 \mathrm{~s}$ the $5^{\prime}-O$-DMTr/2'$O$-Fpmp strategy to RNA synthesis showed great promise (Rao et al., 1993; Capaldi and Reese, 1994). Since that time, however, there have been very few reports of successful RNA syntheses using this protocol, although these monomers are commercially available. The results obtained with the 2'-O-(o-nitrobenzyloxymethyl) (S.21; Schwartz et al., 1992) and 2'-O-(p-nitrobenzyloxymethyl) (S.22; Gough et al., 1996) groups also appeared quite encouraging. Again, since the initial reports describing this chemistry, there have been few follow-ups,
Synthesis of Unmodified Oligonucleotides 
and the use of these $2^{\prime}$-protecting groups does not appear to have gained an appreciable audience beyond its initial developers. Other $2^{\prime}$-protecting groups that, like TBDMS and Fpmp, are compatible with current DNA synthesis protocols are the convertible protecting groups $\mathbf{S . 1 8}$ (Rastogi and Usher, 1995) and S.19 (Pfleiderer et al., 1996). These $2^{\prime}$ - $O$-acetal-derived groups look interesting, but there have been few reports since the initial publications.

Of the synthetic methods that have been designed specifically for RNA synthesis, none is currently commercially available. Around 1990, there were reports citing the combination of $5^{\prime}$ - $O$-Fmoc and either 2'-O-Mthp (Lehmann et al., 1989) or $2^{\prime}$-O-IPE (Ogawa et al., 1991) that provided good-quality oligoribonucleotides; however, the longest oligomer synthesized was a 21-mer. No further communication regarding oligoribonucleotide synthesis with these protecting groups have surfaced. The $5^{\prime}-O$-SIL/2'-O-ACE protocol, however, looks very attractive (Scaringe et al., 1998). The quality of the product is excellent, and oligomers of up to 36 residues have been synthesized. Currently, none of these amidites is commercially available, although efforts are under way to commercialize the $5^{\prime}-O-\mathrm{SIL} / 2^{\prime}-O$-ACE method.

It seems clear that TBDMS chemistry is the current choice for the synthesis of oligoribonucleotides. The amidites are commercially available, and quality products can be produced on a reasonable scale. RNA synthesis chemistry using the 2 '-O-TBDMS group, however, has not yet reached the level achieved by DNA synthesis. As a result, the search for improved protocols or new approaches altogether persists.

\section{LITERATURE CITED}

Andrus, A., Beaucage, S., Ohms, J., and Wert, K. 1986. American Chemical Society Meeting, New York, Organic Division, Abstract 333.

Beaucage, S.L. and Iyer, R.P. 1992. Advances in the synthesis of oligonucleotides by the phosphoramidite approach. Tetrahedron 48:22232311.

Beijer, B., Sulston, I., Sproat, B.S., Rider, P., Lamond, A.I., and Neuner, P. 1990. Synthesis and applications of oligoribonucleotides with selected $2^{\prime}$ - $O$-methylation using the $2^{\prime}-O$ - $[1-(2-$ fluorophenyl)-4-methoxypiperidin-4-yl] protecting group. Nucl. Acids Res. 18:5143-5151.

Bellon, L. 2000. Oligoribonucleotides with 2'-O-(tbutyldimethylsilyl) groups. In Current Protocols in Nucleic Acid Chemistry (S.L. Beaucage, D.E. Bergstrom, G.D. Glick and R.A. Jones, eds.) in press. John Wiley \& Sons, New York.
Bergmann, F. and Pfleiderer, W. 1994a. Solid-phase synthesis of oligoribonucleotides using the 2dansylethoxycarbonyl group for 5'-hydroxy protection. Helvetica Chim. Acta 77:481-500.

Bergmann, F. and Pfleiderer, W. 1994b. The 2-dansylethoxycarbonyl group for the protection of the 5'-hydroxy function in oligoribonucleotide synthesis. Helvetica Chim. Acta 77:989-998.

Capaldi, D.C. and Reese, C.B. 1994. Use of the 1-(2-fluorophenyl)-4-methoxypiperidin-4-yl (Fpmp) and related protecting groups in oligoribonucleotide synthesis: Stability of internucleotide linkages to aqueous acid. Nucl. Acids Res. 22:2209-2216.

Cech, T. 1992. Ribozyme engineering. Curr. Opin. Struct. Biol. 2:605-609.

Chaix, C., Duplaa, A.M., Molko, D., and Téoule, R. 1989. Solid phase synthesis of the 5'-half of the initiator t-RNA from B. subtilis. Nucl. Acids Res. 17:7381-7393.

Christodoulou, C., Agrawal, S., and Gait, M.J. 1986. Incompatibility of acid-labile 2 ' and $5^{\prime}$ protecting groups for solid-phase synthesis of oligoribonucleotides. Tetrahedron Lett. 27:1521-1522.

Cook, K.S., Fisk, G.J., Hauber, J., Usman, N., Daly, T.J., and Rusche, J.R. 1991. Characterization of HIV-1 REV protein: Binding stoichiometry and minimal RNA substrate. Nucl. Acids Res. 19:1577-1583.

Dahl, B.J., Bjergarde, K., Henriksen, L., and Dahl, O. 1990. Deoxyribonucleoside phosphorodithioates. Preparation of dinucleoside phosphorodithioates from nucleoside thiophosphoramidites. Acta Chem. Scand. 44:639-641.

Damha, M.J., Giannaris, P.A., and Zabarylo, S.V. 1990. An improved procedure for derivitization of controlled-pore glass beads for solid-phase oligonucleotide synthesis. Nucl. Acids Res. 18:3813-3820.

deBear, J.S., Hayes, J.A., Koleck, M.P., and Gough, G.R. 1987. A universal glass support for oligonucleotide synthesis. Nucleosides Nucleotides 6:821-830.

Francklyn, C. and Schimmel, P.R. 1989. Aminoacylation of RNA minihelixes with alanine. Nature 337:478-481.

Gait, M.J., Pritchard, C., and Slim, G. 1991. Oligoribonucleotide synthesis. In Oligonucleotides and Analogues, A Practical Approach (F. Eckstein, ed.) pp 25-48. Oxford University Press, Oxford.

Gasparutto, D., Livache, T., Bazin, H., Duplaa, A.M., Guy, A., Khorlin, A., Molko, D., Roget, A., and Téoule, R. 1992. Chemical synthesis of a biologically active natural tRNA with its minor bases. Nucl. Acids Res. 20:5159-5166.

Gold, L. 1988. Posttranscriptional regulatory mechanisms in Escherichia coli. Annu. Rev. Biochem. 57:199-233.

Gough, G.R., Miller, T.J., and Mantick, N.A. 1996. p-Nitrobenzyloxymethyl: A new fluoride-removable protecting group for ribonucleoside 2 hydroxyls. Tetrahedron Lett. 37:981-982. 
Hayakawa, Y., Kataoka, M., and Noyori, R. 1996. Benzimidazolium triflate as an efficient promoter for nucleotide synthesis via the phosphoramidite approach. J. Org. Chem. 61:79967997.

Hayes, J.A., Brunden, M.J., Gilham, P.T. and Gough, G.R. 1985. High-yield synthesis of oligoribonucleotides using o-nitrobenzyl protection of 2'-hydroxyls. Tetrahedron Lett. 26:24072410 .

Himmelsbach, F., Schulz, B.S., Trichtinger, T., Charubala, R., and Pfleiderer, W. 1984. The $p$ nitrophenylethyl (Npe) group. Tetrahedron 40:59-72.

Hogrefe, R.I., McCaffrey, A.P., Borozdina, L.U., McCampbell, E.S., and Vaghefi, M.M. 1994. Effect of excess water on the desilylation of oligoribonucleotides using tetrabutylammonium fluoride. Nucl. Acids Res. 21:4739-4741.

Iwai, S. and Ohtsuka, E. 1988. 5'-Levulinyl and 2 '-tetrahydrofuranyl protection for the synthesis of oligoribonucleotides by the phosphoramidite approach. Nucl. Acids Res. 16:9443-9456.

Iwai, S., Yamada, E., Asaka, M., Hayase,Y., Inoue, H., and Ohtsuka, E. 1987. A new solid-phase synthesis of oligoribonucleotides by the phosphoro-p-anisidate method using tetrahydrofuranyl protection of 2'-hydroxyl groups. Nucl. Acids Res. 15:3761-3772.

Johnson, K.A. and Benkovic, S.J. 1990. Analysis of protein function by mutagenesis. In The Enzymes, Vol. 19 (Sigman, D.S. and Boyer, P.D., eds) pp. 159-211. Academic Press, San Diego.

Karaoglu, D. and Thurlow, D.L. 1991 A chemical interference study on the interaction of ribosomal protein L11 from in Escherichia coli with RNA molecules containing its binding site from 23S rRNA. Nucl. Acids Res. 19:5293-5300.

Klosel, R., Konig, S., Lehnhoff, S., and Karl, R.M. 1996. The 1,1-dianisyl-2,2,2-trichloroethyl group as a 2 -hydroxyl protection of ribonucleotides. Tetrahedron 52:1493-1502.

Lehmann, C., Xu, Y.Z., Christodoulou, C., Tan, Z.K., and Gait, M.J. 1989. Solid-phase synthesis of oligoribonucleotides using 9-fluorenylmethoxycarbonyl (Fmoc) for 5'-hydroxyl protection. Nucl. Acids Res. 17:2379-2390.

Leiber, E. and Enkoju, T. 1961. Synthesis and properties of 5-(substituted) mercaptotetrazoles. $J$. Org. Chem. 26:4472-4479.

Lyttle, M.H., Wright, P.B., Sinha, N.D., Bain, J.D., and Chamberlin, A.R. 1991. New nucleoside phosphoramidites and coupling protocols for solid-phase RNA synthesis. J. Org. Chem. 56:4608-4615.

McCollum, C. and Andrus, A. 1991. An optimized polystyrene support for rapid, efficient oligonucleotide synthesis. Tetrahedron Lett. 32:40694072.
Ogawa, T., Hosaka, H., Makita, T., and Takaku, H. 1991. Solid-phase synthesis of oligoribonucleotides using 5'-9-fluorenylmethoxycarbonyl and 2'-1-(isopropoxyl)ethyl protection. Chem. Lett. 1169-1172.

Ohtsuka, E., Fujiyama, K., and Ikehara, M. 1981. Studies on transfer ribonucleic acids and related compounds. XL. Synthesis of an eicosaribonucleotide corresponding to residues 35-54 of tRNA ${ }^{\text {fMet }}$ from E. coli. Nucl. Acids Res. 9:35033522 .

Pfister, M., Farkas, S., Charubala, R., and Pfleiderer, W. 1988. Recent progress in oligoribonucleotide synthesis. Nucleosides Nucleotides 7:595-600.

Pfleiderer, W., Matysiak, S., Bergmann, F., and Schnell, R. 1996. Recent progress in oligonucleotide synthesis. Acta Biochim. Polonica 43:37-44.

Pon, R.T., Usman, N., and Ogilvie, K.K. 1988. Derivitization of controlled pore glass beads for solid phase oligonucleotide synthesis. BioTechniques 6:768-774.

Rao, M.V., Reese, C.B., Schehlmann, V., and Yu, P.S. 1993. Use of the 1-(2-fluorophenyl)-4methoxypiperidin-4-yl (Fpmp) protecting group in the solid-phase synthesis of oligo- and polyribonucleotides. J. Chem. Soc. Perkin Trans. I:43-55.

Rastogi, J. and Usher, D. 1995. A new 2'-hydroxyl protecting group for the automated synthesis of oligoribonucleotides. Nucleic Acids Res. 23:4872-4877.

Reddy, M.P., Hanna, N.B., and Farooqui, F. 1994. Fast cleavage and deprotection of oligonucleotides. Tetrahedron Lett. 35:4311-4314.

Reddy, M.P., Hanna, N.B., and Farooqui, F. 1995. Methylamine deprotection provides increased yield of oligoribonucleotides. Tetrahedron Lett. 36:8929-8932.

Reese, C.B. and Skone, P.A. 1985. Action of acid on oligoribonucleotide phosphotriester intermediates. Effect of released vicinal hydroxy functions. Nucl. Acids Res. 13:3501-.

Reese, C.B., Serafinowska, H.T., and Zappia, G. 1986. An acetal group suitable for the protection of 2 -hydroxy functions in rapid oligoribonucleotide synthesis. Tetrahedron Lett. 27:22912294.

Sakatsume, O., Yamaguchi, T., Ishikawa, M., Hirao, I., Miura, K., and Takaku, H. 1991a. Solid phase synthesis of oligoribonucleotides by the phosphoramidite approach using 2'-O-1-(2-chloroethoxy)ethyl protection. Tetrahedron 47:8717-8728.

Sakatsume, O., Ogawa, T., Hosaka, H., Kawashima, M., Takaki, M., and Takaku, H. 1991b. Synthesis and properties of non-hammerhead RNA using 1-(2-chloroethoxy)ethyl group for the protection of 2'-hydroxyl function. Nucleosides Nucleotides 10:141-153.
Synthesis of Unmodified Oligonucleotides 
Scaringe, S.A., Francklyn, C., and Usman, N. 1990. Chemical synthesis of biologically active oligoribonucleotides using $\beta$-cyanoethyl protected ribonucleoside phosphoramidites. Nucl. Acids Res. 18:5433-5341.

Scaringe, S.A., Wincott, F.E., and Caruthers, M.H. 1998. Novel RNA synthesis method using 5'-Osilyl-2'-O-orthoester protecting groups. J. Am. Chem Soc. 120:11820-11821.

Schwartz, M.E., Breaker, R.R., Asteriadis, G.T., deBear, J.S., and Gough, G.R. 1992. Rapid synthesis of oligoribonucleotides using $2^{\prime}-O$ - $(o-n i-$ trobenzyloxymethyl)-protected monomers. Bioorg. Med. Chem. Lett. 2:1019-1024.

Sinha, N.D., Davis, P., Usman, N., Pérez, J., Hodge, R., Kremsky, J., and Casale, R. 1993. Labile exocyclic amine protection in DNA, RNA and oligonucleotide analog synthesis facilitating $\mathrm{N}$ deacylation, minimizing depurination and chain degradation. Biochimie 75:13-23.

Sproat, B., Colonna, F., Mullah, B., Tsou, D., Andrus, A., Hampel, A., and Vinayak, R. 1995. An efficient method for the isolation and purification of oligoribonucleotides. Nucleosides Nucleotides 14:255-273.

Tanaka, T., Tamatsukuri, S., and Ikehara, M. 1986. Solid phase synthesis of oligoribonucleotides using o-nitrobenzyl protection of 2'-hydroxyl via a phosphite triester approach. Nucl. Acids Res. 14:6265-6279.

Theisen, P., McCollum, C., and Andrus, A. 1993. N-6-Dialkylformamidine-2'-deoxyadenosine phosphoramidites in oligodeoxynucleotide synthesis. Rapid deprotection of oligodeoxynucleotides. Nucleosides Nucleotides 12:10331046.

Usman, N., Pon, R.T., and Ogilvie, K.K. 1985. Preparation of ribonucleoside 3 '- $O$-phosphoramidites and their application to the automated solid phase synthesis of oligonucleotides. Tetrahedron Lett. 26:4567-4570.
Usman, N., Ogilvie, K.K., Jiang, M.-Y., and Cedergren, R.J. 1987. Automated chemical synthesis of long oligoribonucleotides using 2'-O-silylated ribonucleoside $3^{\prime}$ - $O$-phosphoramidites on a controlled-pore glass support: Synthesis of a 43-nucleotide sequence similar to the 3'-half molecule of an in Escherichia coli formylmethionine tRNA. J. Am. Chem. Soc. 109:78457854.

Vargeese, C., Carter, J., Yegge, J., Krivjansky, S., Settle, A., Kropp, E., Peterson, K., and Pieken, W. 1998. Efficient activation of nucleoside phosphoramidites with 4,5-dicyanoimidazole during oligonucleotide synthesis. Nucl. Acids Res. 26:1046-1050.

Vinayak, R., Ratmeyer, L., Wright, P., Andrus, A., and Wilson, D. 1994. Chemical synthesis of biologically active RNA using labile protecting groups. In Innovations and Perspectives in Solid Phase Synthesis (R. Epton, ed.) pp 45-50. Mayflower Worldwide, Birmingham.

Westman, E. and Strömberg, R. 1994. Removal of $t$-butyldimethylsilyl protection in RNA-synthesis. Triethylamine trihydrofluoride (TEA, 3HF) is a more reliable alternative to tetrabutylammonium fluoride (TBAF). Nucl. Acids Res. 22:2430-2431.

Wincott, F., DiRenzo, A., Shaffer, C., Grimm, S., Tracz, D., Workman, C., Sweedler, D., Gonzalez, C., Scaringe, S., and Usman, N. 1995. Synthesis, deprotection, analysis and purification of RNA and ribozymes. Nucl. Acids Res. 23:2677-2684.

Wu, T., Ogilvie, K.K., and Pon, R.T. 1988. N-Phenoxyacetylated guanosine and adenosine phosphoramidites in the solid phase synthesis of oligoribonucleotides: Synthesis of a ribozyme sequence. Tetrahedron Lett. 34:4249-4252.

\section{Contributed by Francine E. Wincott Ribozyme Pharmaceuticals, Inc. Boulder, Colorado}

Strategies for Oligoribonucleotide Synthesis According to the Phosphoramidite Method 